\title{
Saúde Bucal em sistemas universais de saúde: análise comparativa do Brasil e Reino Unido
}

\author{
Oral Health in universal health systems: comparative analysis of Brazil and the United Kingdom \\ Salud Bucal en sistemas universales de salud: análisis comparativo de Brasil y Reino Unido
}

Recebido: 19/07/2021 | Revisado: 29/07/2021 | Aceito: 02/08/2021 | Publicado: 08/08/2021

\author{
Ana Maria Freire de Souza Lima \\ ORCID: https://orcid.org/0000-0002-9285-194X \\ Universidade Federal do Recôncavo da Bahia, Brasil \\ E-mail: anamariafreire@ufrb.edu.br \\ Larissa de Sousa Lyra \\ ORCID: https://orcid.org/0000-0001-6597-3215 \\ Universidade Federal do Recôncavo da Bahia, Brasil \\ E-mail: larissalyra@aluno.ufrb.edu.br \\ Larissa Lima Viana Guimarães \\ ORCID: https://orcid.org/0000-0001-5483-5538 \\ Universidade Federal do Recôncavo da Bahia, Brasil \\ E-mail: larissa.limavg@hotmail.com \\ Michele Pontes Teixeira \\ ORCID: https://orcid.org/0000-0001-5089-4469 \\ Universidade Federal do Recôncavo da Bahia, Brasil \\ E-mail: pontesmichele96@gmail.com \\ Thaís Regis Aranha Rossi \\ ORCID: https://orcid.org/0000-0002-2561-088X \\ Universidade do Estado da Bahia, Brasil \\ E-mail: thais.aranha@gmail.com \\ Sônia Cristina Lima Chaves \\ ORCID: https://orcid.org/0000-0002-1476-8649 \\ Universidade Federal da Bahia, Brasil \\ E-mail: schaves@ufba.br
}

\begin{abstract}
Resumo
Este estudo analisou as principais características de dois sistemas universais, o National Health Service (NHS), no Reino Unido, e o Sistema Único de Saúde (SUS), no Brasil, em relação às ações e serviços de atenção à saúde bucal. Foi realizada revisão da literatura, análise documental e de dados secundários a partir de bases oficiais do Brasil e do Reino Unido. Observaram-se diferenças no financiamento, população coberta, infraestrutura, organização, prestação e gestão das ações e serviços. Os indicadores sociodemográficos e de condições de saúde bucal são mais desfavoráveis na situação brasileira, contudo, as desigualdades persistem também no Reino Unido. Nos dois países, a saúde é vista como direito constitucional, entretanto as ações de saúde bucal do NHS são restritas a um público específico e ofertada por profissionais do setor privado, com grande parte do custeio de procedimentos pelas famílias. As sucessivas reformas do NHS contribuíram para restrição do acesso. Já o SUS apresenta serviços de saúde bucal, de atenção primária e especializada, de oferta universal e pública, ainda que a cobertura seja insuficiente e com problemas na implantação. Medidas de austeridade foram identificadas como ameaça aos princípios da universalidade e integralidade nos dois países. A análise comparativa auxilia na identificação de elementos que contribuem para o avanço da oferta pública da atenção odontológica.
\end{abstract}

Palavras-chave: Sistemas de saúde; Política de saúde; Sistema Único de Saúde; Saúde bucal; Sistemas nacionais de Saúde.

\begin{abstract}
This study analyzed the main characteristics of two universal systems, the National Health Service (NHS), in the United Kingdom, and the Unified Health System (SUS), in Brazil, in relation to oral health care actions and services. A literature review, document and secondary data analysis were performed from official databases in Brazil and the United Kingdom. There were differences in financing, covered population, infrastructure, organization, provision and management of actions and services. The sociodemographic and oral health indicators are more unfavorable in the Brazilian situation, however, inequalities persist also in the United Kingdom. In both countries, health is seen as a constitutional right, but NHS oral health actions are restricted to a specific public and offered by professionals from the private sector, with a large part of the cost of procedures by families. Successive reforms of the NHS have contributed to restricting access. The SUS, on the other hand, presents oral health services, primary and specialized care, universal
\end{abstract}


and public offer, even if the coverage is insufficient and with problems in implementation. Austerity measures have been identified as a threat to the principles of universality and integrality in both countries. Comparative analysis helps in the identification of elements that contribute to the advancement of public provision of dental care.

Keywords: Health systems; Health policy; Unified Health System; Oral health; National health systems.

\section{Resumen}

Este estudio analizó las principales características de dos sistemas universales, el National Health Service (NHS), en el Reino Unido, y el Sistema Único de Salud (SUS), en Brasil, en relación a las acciones y servicios de atención a la salud bucal. Se realizó revisión de la literatura, análisis documental y de datos secundarios a partir de bases oficiales de Brasil y del Reino Unido. Se observaron diferencias en el financiamiento, población cubierta, infraestructura, organización, prestación y gestión de las acciones y servicios. Los indicadores sociodemográficos y de condiciones de salud bucal son más desfavorables en la situación brasileña, sin embargo, las desigualdades persisten también en el Reino Unido. En los dos países, la salud es vista como derecho constitucional, sin embargo las acciones de salud bucal del NHS son restringidas a un público específico y ofertada por profesionales del sector privado, con gran parte del costeo de procedimientos por las familias. Las sucesivas reformas del NHS contribuyeron a restringir el acceso. Ya el SUS presenta servicios de salud bucal, de atención primaria y especializada, de oferta universal y pública, aunque la cobertura sea insuficiente y con problemas en la implantación. Las medidas de austeridad han sido identificadas como una amenaza a los principios de universalidad e integridad en ambos países. El análisis comparativo auxilia en la identificación de elementos que contribuyen para el avance de la oferta pública de la atención odontológica.

Palabras clave: Sistemas de salud; Política de salud; Sistema Único de Salud; Salud oral; Sistemas nacionales de salud.

\section{Introdução}

As doenças bucais são um problema de saúde pública em escala mundial, impactando na saúde e na qualidade de vida das pessoas, sendo crianças, idosos e grupos em situação de pobreza os mais afetados (Peres et al., 2019). No Reino Unido (RU), o National Health Service (NHS) surgiu em 1948, baseado nos princípios de equidade e integralidade e com cobertura universal gratuita, à exceção de alguns tratamentos, dentre eles o odontológico (Giovanella \& Stegmüller, 2014; Tanaka \& Oliveira, 2007). O seguro privado é usado para atendimentos não cobertos pelo NHS, para acessar leitos privados em hospitais públicos ou serviços em hospitais privados, estes últimos procurados pela população de maior renda (Ferreira \& Mendes, 2018).

O NHS foi criado logo após a implantação da política de bem-estar social, Welfare State, em 1945, com princípios de proteção social e prestação de serviços a toda população, incluindo saúde, educação e habitação (Ferreira \& Mendes, 2018; Giovanella \& Stegmüller, 2014). Em 1991, o NHS passou por reforma no âmbito de um governo mais conservador, desenvolvendo uma lógica de mercado interno com competição, ainda que regulada, e manteve a atenção primária como porta de entrada prioritária (Tanaka \& Oliveira, 2007). Em 1997, um novo governo de orientação partidária ideológica de esquerda realizou a chamada "reforma da reforma", com fomento à avaliação de desempenho e regionalização da atenção em saúde (Giovanella \& Stegmüller, 2014; Tanaka \& Oliveira, 2007). Reformas mais recentes acentuaram a progressiva liberalização e fechamento de serviços, ampliando a restrição de acesso (Filippon et al., 2016). A maior parte do financiamento do sistema britânico é público, originado principalmente dos impostos e do sistema de Seguridade Social, semelhante ao financiamento do Sistema Único de Saúde (SUS) no Brasil (Giovanella \& Stegmüller, 2014; Tanaka \& Oliveira, 2007).

O SUS foi inserido na Constituição Federal de 1988, após um processo de redemocratização política, com participação de diferentes atores da sociedade no que ficou conhecido como Movimento da Reforma Sanitária (Santos, 2018). Apesar de ter caráter universal, público e prever a oferta de ações e serviços de saúde em todos os níveis de complexidade, o financiamento insuficiente desde o início da sua implantação limitou a expansão e a capacidade instalada para atender a esses princípios, e, em paralelo, houve livre crescimento do setor privado, o qual deveria ser apenas de natureza complementar, mas detém hoje grande parte dos serviços de diagnóstico, bem como tratamentos de média e alta complexidade (Paim, 2018; Santos, 2018).

Há uma contínua negligência na saúde bucal na política global de saúde, e, na maioria dos países, os serviços públicos são restritos a grupos específicos (Peres et al., 2019; Pucca et al., 2015). O NHS tem sido referência para muitas análises comparativas, com destaque para uma série mais recente de estudos em nove países da União Europeia, e em todos a saúde bucal 
continua predominantemente fora da cobertura da oferta pública (Sinclair et al., 2019). Nesse aspecto, o Brasil se diferencia por oferecer no âmbito do SUS, sobretudo a partir de 2004, serviços públicos odontológicos de atenção primária e especializada (Pucca et al., 2015). O sistema britânico, mesmo tendo sido criado 40 anos antes, e em alguma medida ser uma das referências para o SUS, não apresenta provisão de serviços de saúde bucal de forma universal, além de ter passado por reformas que o deixam cada vez mais restritivo (Filippon et al., 2016). Nesse sentido, o presente estudo buscou atualizar e discutir as principais características dos dois sistemas, NHS e SUS, em relação às ações de atenção à saúde bucal.

\section{Metodologia}

Estudo de análise comparada de dois casos sobre a atenção à saúde bucal de oferta pública em sistemas universais de saúde. Foram analisados os casos do Sistema Único de Saúde (SUS) no Brasil e do National Health Service (NHS) no Reino Unido, através de revisão da literatura nas bases de dados Medline, LILACS, SciELO, PubMed e EBSCOhost, e consulta a documentos e dados disponíveis em sites oficiais e institutos nacionais de estatística, a saber: Organização Mundial de Saúde (OMS), Ministério da Saúde do Brasil (MS), United Kingdom Department of Health e National Health Service (NHS).

A revisão integrativa adaptada de Whittemore e Knalf (2005) foi estruturada em cinco etapas: (i) identificação do problema; (ii) busca na literatura; (iii) seleção e extração dos dados; (iv) análise dos dados e (v) apresentação dos resultados. Utilizaram-se os descritores "Health Policy"; “Oral Health"; "Universal Health Systems”; Unified Health System”; "National Health Service"; "United Kingdom" e "Brazil”. Os critérios de inclusão foram: artigos científicos disponíveis em texto completo, nas línguas portuguesa e inglesa, com informações sobre a atenção à saúde bucal de oferta pública no âmbito do SUS e do NHS, no período de 2000 a 2020, considerando os seguintes componentes e categorias de análise, adaptados de Souza e Bahia (2014): a) população, b) infraestrutura, c) organização e prestação de serviços, d) financiamento e gestão, detalhados no Quadro 1 .

Quadro 1. Componentes do sistema de saúde, no que se refere à atenção à saúde bucal, analisados neste estudo.

\begin{tabular}{|l|l|l|}
\hline Item & Componente & Detalhamento \\
\hline 1 & População & $\begin{array}{l}\text { Componente mais importante de um sistema de saúde. Reflete a capacidade dos serviços de saúde em resolver } \\
\text { problemas de saúde dos indivíduos e coletividade. Foi considerada a população que pode acessar o serviço } \\
\text { de forma gratuita e indicadores de saúde bucal. }\end{array}$ \\
\hline 2 & $\begin{array}{l}\text { Trabalhadores, estabelecimentos de atenção primária e especializada, equipamentos, insumos e } \\
\text { conhecimento. }\end{array}$ \\
\hline 3 & Organização e prestação de serviços & $\begin{array}{l}\text { A organização se refere à disposição dos recursos humanos e tecnológicos para ações de saúde. A prestação } \\
\text { de serviços envolve as ações finalísticas do sistema de saúde (ações de promoção, prevenção, diagnóstico e } \\
\text { tratamento de doenças e agravos, e de reabilitação). }\end{array}$ \\
\hline 4 & Financiamento e gestão & $\begin{array}{l}\text { Aporte de recursos financeiros aos sistemas de saúde e aplicação desses recursos. A gestão se refere desde a } \\
\text { elaboração de políticas até a execução das ações. Foi incluída a forma de remuneração dos profissionais. }\end{array}$ \\
\hline
\end{tabular}

Fonte: Adaptado de Souza e Bahia (2014).

\section{Resultados e Discussão}

A determinação social na saúde a partir do recorte da saúde bucal, está bem documentada (Hui et al., 2020; Peres et al., 2019; Watt et al., 2019). Os piores indicadores sociodemográficos brasileiros corroboram para maior prevalência de agravos bucais na população, mesmo com a atualização da resposta estatal em 2004, através da Política Nacional de Saúde Bucal, intitulada à época "Programa Brasil Sorridente", que promoveu expansão das ações e implantação de novos serviços (Pucca et al., 2015). O Quadro 2 apresenta uma síntese dos principais indicadores sociodemográficos e informações coletadas sobre a saúde bucal no Reino Unido (RU) e no Brasil, com foco no NHS e no SUS, respectivamente. A seguir, discutem-se as principais características da atenção à saúde bucal nos dois sistemas universais analisados, a partir dos componentes de um sistema de serviços de saúde adaptados de Souza e Bahia (2014). 
Com relação a população coberta, no NHS atualmente o atendimento odontológico é gratuito para menores de 18 anos, estudantes em período integral de até 19 anos, gestantes ou mulheres que tiveram filhos nos últimos 12 meses, pacientes no período do internamento hospitalar, e beneficiários de auxílio social e seus dependentes menores de 20 anos (NHS, 2021). O restante da população custeia os serviços em três faixas que variam conforme a cobertura, sendo o valor da faixa $1 £ 23,80$ (R\$ $164,32)$, da faixa $2, £ 65,20(\mathrm{R} \$ 450,15)$, e a faixa 3 que custa $£ 282,80$ ( $\mathrm{R} \$ 1.952,50)$ (NHS, 2021). Os valores foram convertidos pelo Conversor de Moedas do Banco Central do Brasil em 30/06/2021, quando 1 libra esterlina custava 6,9 reais, e referem-se ao tratamento e não à quantidade de idas ao profissional. Procedimentos estéticos não estão incluídos (NHS, 2020). Existem serviços odontológicos comunitários em clínicas, casas de repouso e escolas (Cylus et al., 2015).

Sobre a prevalência de cárie, houve melhora no RU, comparando os anos de 1973, quando havia 97\% da população afetada, com o ano 2003, reduzindo para 49\% (Chestnutt, 2013). As crianças aos 12 anos têm em média menos de um dente cariado ou extraído, sendo que os níveis de cárie dentária no período entre 2003 e 2013 reduziram em todas as faixas etárias (5, 8, 12, e 15 anos) (Downer et al., 2005; Masood et al., 2019). A perda dentária total em adultos também reduziu, chegando a 6\%, em 2009, com 86\% dos adultos com mais de 21 dentes (Steele et al., 2012). Todavia, as desigualdades em saúde bucal permanecem, e há maior concentração da doença na população de baixa renda, incluindo crianças (Chestnutt, 2013; Masood et al., 2019).

No Brasil, em 2003, a proporção de crianças livres de cárie aos 5 e 12 anos no Brasil, eram respectivamente de 40,62\% e 31,08\%, e a perda dentária era de 65,72\% na faixa etária de 35-44 anos, e 92,95\% entre idosos de 65-74 anos (Roncalli et al., 2012). Em 2010, os dados apontaram redução da prevalência de cárie, aumentando para 46,6\% e 43,5\% a porcentagem de crianças livres da doença respectivamente aos 5 e aos 12 anos, com permanência de alto índice de perda dentária na população adulta e idosa (Brasil, 2012) (Quadro 2). A meta da OMS de CPOD aos 12 anos (<1,0) não foi alcançada pelo Brasil, enquanto que o Reino Unido cumpriu, e já se aproxima da meta de 80\% das crianças livres de cárie que tinha sido estabelecida para 2020 (Hobdell et al., 2003). Os problemas de saúde bucal se mantêm como uma questão a ser enfrentada em todos os países, principalmente nos de baixa e média renda (Peres et al., 2019).

Em relação aos trabalhadores, no RU há maior diversidade de tipos de profissionais da odontologia quando comparada ao Brasil (Quadro 2). A maioria dos 119,3 mil registrados são enfermeiras dentais/dental nurses (49\%), seguidas de dentistas (36\%), com uma proporção de 6,3 dentistas para cada 10.000 habitantes (GDC, 2019). A média da União Europeia é de 5 cirurgiões-dentistas/10.000 hab. (WHO, 2020). São cerca de 10\% registrados como especialistas e existem 13 especialidades odontológicas reconhecidas (GDC, 2019). O número de dentistas aumentou, com uma taxa média de crescimento anual de 2,1\% entre 2007 e 2013, mas ainda é inferior ao de países comparáveis (Cylus et al., 2015). De 2000 até 2019 o aumento foi de 36\% ( $\mathrm{n}=11.144)$, com 28\% formados fora do Reino Unido (GDC, 2019; Davda et al., 2020).

Quadro 2. Indicadores sociodemográficos e informações sobre o sistema de saúde e a saúde bucal no Brasil/SUS e no Reino Unido/NHS.

\begin{tabular}{|l|l|l|}
\hline \multicolumn{1}{|c|}{ Indicadores } & \multicolumn{1}{c|}{ Brasil/ SUS } & \multicolumn{1}{c|}{ Reino Unido/ NHS } \\
\hline $\begin{array}{l}\text { Indicadores } \\
\text { sociodemográficos }\end{array}$ & $\begin{array}{l}\text { População (2020): 212,5 milhões hab. } \\
\text { PIB (2019): 1,84 trilhão de dólares } \\
\text { PIB per capita (2019): 8,75 dólares } \\
\text { Esperança de vida ao nascer (2019): 75,9 anos } \\
\text { Gastos públicos com saúde (2018): 3,96\% do PIB } \\
\text { IDH (2019): 0,765 }\end{array}$ & $\begin{array}{l}\text { População (2020): 67,5 milhões hab. } \\
\text { PIB (2019): 2,8 trilhões de dólares } \\
\text { PIB per capita (2019): 41,85 dólares } \\
\text { Esperança de vida ao nascer (2019): 81,3 anos } \\
\text { Gastos públicos com saúde (2018): 7,85\% do PIB } \\
\text { IDH (2019): 0,93 }\end{array}$ \\
\hline $\begin{array}{l}\text { Indicadores de saúde } \\
\text { bucal }\end{array}$ & $\begin{array}{l}\text { 2003: Índice CPOD aos 12 anos: 2,8; } \\
\text { 40,62\% e 31,08\%, crianças livres de cárie aos 5 e 12 anos } \\
\text { respectivamente; }\end{array}$ & $\begin{array}{l}\text { 2005: Média de menos de um dente cariado ou extraído } \\
\text { 2012: 67\% das crianças de 5 anos de idade na Escócia não } \\
\text { tiveram experiência de cárie dentária óbvia }\end{array}$ \\
\hline
\end{tabular}




\begin{tabular}{|c|c|c|}
\hline & $\begin{array}{l}27 \% \text { dos adolescentes necessitavam de algum tipo de } \\
\text { prótese dentária; } 4,4 \% \text { dos adultos necessitavam de prótese } \\
\text { total em pelo menos um maxilar; } 24 \% \text { dos idosos } \\
\text { necessitavam de prótese total em pelo menos um maxilar e } \\
16 \% \text { necessitavam de prótese total dupla. } \\
\text { 2010: Índice CPOD aos } 12 \text { anos: } 2,1 \\
46,6 \% \text { e } 43,5 \% \text { crianças livres de cárie aos } 5 \text { e aos } 12 \text { anos, } \\
\text { respectivamente; } \\
13 \% \text { dos adolescentes necessitavam de próteses dentárias } \\
\text { parciais; } 1,3 \% \text { dos adultos necessitavam de prótese total } \\
\text { em pelo menos um maxilar; } 23 \% \text { dos idosos necessitam de } \\
\text { prótese total em pelo menos um maxilar e } 15 \% \text { necessitam } \\
\text { de prótese total dupla. } \\
\text { 2013: } \% \text { de maiores de } 18 \text { anos que perderam todos os } \\
\text { dentes: } 11 \% \text { (16 milhões de hab.) }\end{array}$ & $\begin{array}{l}\text { 2009: } 6 \% \text { da população (Inglaterra, País de Gales e Irlanda } \\
\text { do Norte) com perda dentária total, e a proporção de adultos } \\
\text { com mais de } 21 \text { dentes era de } 86 \% \text {. } \\
\text { 2016: Índice CPOD aos } 12 \text { anos: } 0,8 \\
\text { 2019: } 76,6 \% \text { das crianças de } 5 \text { anos de idade na Inglaterra } \\
\text { não tiveram experiência de cárie dentária óbvia }\end{array}$ \\
\hline Profissionais ${ }^{\mathrm{e}}$ & $\begin{array}{l}\text { 2021: Cirurgiões-Dentistas: } 334.747 \\
\text { 15,6 cirurgiões dentistas/ } 10.000 \text { hab. } \\
\text { Técnicos em Saúde Bucal: } 33.077 \\
\text { Auxiliares em Saúde Bucal: } 146.544 \\
\text { Técnicos em Prótese Dentária: } 23.496 \\
\text { Auxiliares em Prótese Dentária: } 6.554 \\
\text { 2018: } 58.240 \text { cirurgiões-dentistas vinculados ao serviço } \\
\text { público no CNES* } \\
\text { 2020: } 462 \text { faculdades de Odontologia (> } 70 \% \text { privadas) } \\
\text { Duração mínima do curso: } 5 \text { anos }\end{array}$ & $\begin{array}{l}\text { 2019: Cirurgiões-dentistas: } 42.470 \\
6,3 \text { cirurgiões-dentistas/10.000 hab. } \\
\text { Dental nurses: } 58.898 \\
\text { Dental hygienists: } 7.563 \\
\text { Dental therapists: } 3.620 \\
\text { Dental technicians: } 5.776 \\
\text { Orthodontic therapists: } 695 \\
\text { Clinical dental technician: } 375 \\
\text { 2019/2020: } 24.684 \text { cirurgiões-dentistas realizaram atividades } \\
\text { no NHS inglês } \\
\text { 2020: } 18 \text { faculdades de Odontologia (12 na Inglaterra) } \\
\text { Duração mínima do curso: } 5 \text { anos }\end{array}$ \\
\hline $\begin{array}{l}\text { Serviços ofertados e } \\
\text { utilização }^{\mathbf{d}}\end{array}$ & $\begin{array}{l}\text { 2013: \% de pessoas que consultaram dentista nos últimos } \\
12 \text { meses: } 44,4 \% \text { ( } 89,1 \text { milhões de hab.); } \\
\text { 2013: 25,7\% das consultas ao dentista nos últimos } 12 \\
\text { meses foram no SUS, e } 19,6 \% \text { em unidades de atenção } \\
\text { primária. } \\
\text { 2020: Equipes de Saúde Bucal na Atenção Primária: } \\
\text { 28.991; Cobertura populacional 53\%; } \\
\text { 2017: Cobertura de fluoretação das águas de abastecimento } \\
\text { público: } 76,3 \% \text { dos hab. }\end{array}$ & $\begin{array}{l}\text { 2013: \% de crianças aos } 12 \text { anos que consultaram dentista } \\
\text { nos últimos } 12 \text { meses: } \\
\text { Inglaterra: 90\%; País de Gales: 89\%; Irlanda do Norte: } 97 \% \\
\text { 2013: mais de } 85 \% \text { dos consultórios recebem alguma receita } \\
\text { do NHS } \\
\text { \% de crianças atendidas no NHs inglês nos últimos } 12 \text { meses: } \\
\text { 68,7\% (2014); 58,6\% (2018); } \\
\% \text { dos adultos que consultaram um dentista do NHS inglês nos } \\
\text { últimos } 24 \text { meses: } \\
\text { 52\% (2014); 50,7\% (2018); } \\
\text { 2020: Cobertura de fluoretação das águas de abastecimento } \\
\text { público: } 11 \% \text { dos hab., e ocorre apenas na Inglaterra }\end{array}$ \\
\hline
\end{tabular}

hab. = habitantes *Estimativa a partir dos Cadastro Nacional dos Estabelecimentos de Saúde (CNES/ Ministério da Saúde)

Fontes: a: WHO (2018); IBGE (2021); b: Roncalli et al. (2012); Ministério da Saúde (2012); IBGE (2013); Downer et al. (2005); Steele et al. (2012); Watt et al., 2013; Sinclair et al. (2019); NHS (2021); c: Conselho Federal de Odontologia (2021); Gabriel et al. (2020); World Health Organization (2020); General Dental Council (2019); Neumann e Quiñonez (2014); Cylus et al. (2015); Gallagher e Eaton (2015); Davda et al. (2020); Morita et al., 2021 d: Ministério da Saúde (2020); Herkratz et al. (2016); Oral Health Foundation (2020); UK Parliament (2020).

Profissionais vindos da União Europeia e do Espaço Econômico Europeu realizam cerca de 22\% das atividades do NHS (Coughlan \& Shah, 2020). A participação de profissionais estrangeiros no NHS na medicina e enfermagem também ocorre, e está melhor compreendida do que na odontologia (Davda et al., 2020). Dados do Conselho Geral de Odontologia/General Dental Council (GDC) apontam que 32\% desse grupo tem intenção de deixar o RU, principalmente devido à desvalorização da libra em relação ao euro e insatisfações com as condições de trabalho no NHS. Há uma preocupação do órgão de que o Brexit piore a situação de provimento de dentistas no país (Coughlan \& Shah, 2020; Davda et al., 2020)

O escopo de práticas das categorias profissionais da odontologia do RU é estabelecido pelo GDC. As enfermeiras dentais/dental nurses fornecem suporte ao dentista ou outro profissional da área, sendo sua função mais próxima ao auxiliar de saúde bucal (ASB) brasileiro, e podem ter suas ações ampliadas sob supervisão do dentista. Os higienistas/hygienists trabalham mais com prevenção e tratamento da doença periodontal, enquanto os terapeutas dentários/dental therapists realizam alguns tipos de tratamento odontológico direto ao paciente, incluindo anamnese, exames clínicos e restaurações dentárias (GDC, 2013). Ambos possuem mais funções que os técnicos em saúde bucal (TSB) no Brasil (Brasil, 2008). O técnico de clínica 
dentária/clinical dental technician teve seu exercício profissional reconhecido em 2008, e sua prática inclui a prestação de cuidados completos em casos de próteses totais, além de fornecer próteses parciais removíveis mediante prescrição do dentista (GDC, 2013). Este último foi pouco incorporado pelo NHS, seja pela oposição dos dentistas ou pela baixa remuneração (Leyssen et al., 2013).

No Brasil, são atualmente 334.747 dentistas registrados no Conselho Federal de Odontologia (CFO), e uma proporção de 15,6 profissionais para cada 10.000 habitantes (CFO, 2021). Em 2018, 48\% dos dentistas do Cadastro Nacional dos Estabelecimentos de Saúde ( $\mathrm{n}=58.240$ ) atendiam no SUS (Gabriel et al., 2020). Os vínculos trabalhistas no sistema público são diversos e predominam contratos temporários, precarizados e de baixa remuneração, com grande variação do salário médio do cirurgião-dentista na atenção primária, entre 1,05 a 12,67 salários mínimos, e nos centros de especialidades de 3,35 a 7,05 (Gabriel et al., 2020). As profissões técnicas e auxiliares em saúde bucal são regulamentadas pelo CFO e pela Lei Federal 11.889/2008 (Brasil, 2008). O TSB tem um espectro de atividades autorizadas sob supervisão do dentista, porém bem mais reduzido quando comparado a outras profissões técnicas do RU e de outros países (Lenza et al., 2019).

Apesar da legislação e da política nacional de saúde bucal vigente prever e incentivar equipes de saúde bucal no SUS com TSB, essa incorporação foi baixa e muitas vezes o técnico exerce apenas atividades de auxiliar, situação comum também no setor privado (Warmling et al., 2016). Nos dois países há disputas políticas, administrativas, mercadológicas e ideológicas sobre a divisão do trabalho odontológico, sendo que no Brasil o grande quantitativo de dentistas agrava a competição, em detrimento da cooperação ou complementação das ações (Csikar et al., 2009; Leyssen et al., 2013; Zanetti et al., 2012). Cabe destacar ainda que o GCD, apesar de financiado pelos profissionais da odontologia, é um órgão regulador composto também por membros leigos, sob controle da The Professional Standards Authority, uma autoridade reguladora nacional subordinada ao Parlamento do Reino Unido, enquanto que no Brasil o CFO é uma autarquia autorregulada exclusivamente por cirurgiõesdentistas (CFO, 2020; Gallagher et al., 2015).

Um elemento importante desse cenário é a regulação da formação de cirurgiões-dentistas. No RU, todas as faculdades de odontologia são administradas pelo Estado e existe um sistema de controle para o ingresso no curso, com decisões sobre a cobrança de taxas tomadas em nível nacional. Na Inglaterra, onde se concentram a maioria das escolas, as taxas foram introduzidas antes dos anos 2000 e são cobradas depois que o estudante começa a trabalhar. No País de Gales e Irlanda do Norte os valores são menores, e na Escócia, o primeiro diploma não é cobrado (Gallagher et al., 2015). Já no Brasil, são 462 faculdades em funcionamento, quase $80 \%$ privadas. O número de vagas e abertura de novas escolas é regulado de forma frágil pelo Ministério da Educação, com forte influência do mercado privado, e, a rápida expansão dos cursos vem agravando a distribuição regional desigual dos profissionais (Morita et al., 2021). O governo federal possui dois programas de financiamento para vagas nas faculdades particulares, o ProUni e o Fies. O ProUni destina bolsas de estudos parciais e totais em troca de isenção de impostos federais, e no Fies o estudante paga o valor financiado com a participação dos bancos, após a inserção no mercado de trabalho (Miranda \& Azevedo, 2020).

Quanto à infraestrutura e organização dos serviços, cabe destacar que apesar do acesso ao SUS ser universal e gratuito para todos os cidadãos, a oferta de serviços é historicamente insuficiente. A cobertura das equipes de saúde bucal na atenção primária é de 53\%, e são aproximadamente 1.100 Centros de Especialidades Odontológicas (CEO), o que representa uma proporção de um CEO para cada 217.596 habitantes, com maior concentração nas regiões Nordeste e Sudeste (Brasil, 2020; Herkratz et al., 2016). Cerca de $12 \%$ da população possui plano privado exclusivamente odontológico, com crescimento do número de usuários de 2,6 milhões em 2000 para 24,3 milhões em 2018, sendo que a convivência entre os sistemas público e privado é pouco regulada e com subsídios fiscais que favorecem o setor privado (Rossi et al., 2019).

No SUS, os serviços são de oferta pública, divididos em atenção primária à saúde (APS), oferecidos pelas equipes de saúde bucal (1 dentista e 1 ASB e/ou TSB) vinculadas a Estratégia de Saúde da Família (ESF), e atenção especializada, oferecidos 
pelo CEO, que podem conter as especialidades de Endodontia, Periodontia, Prótese Dentária, Cirurgia Oral, Estomatologia, Odontologia para Pacientes com Necessidades Especiais, Ortodontia e Implantodontia (Cayetano et al., 2019; Pucca et al., 2015). Ambos serviços recebem um repasse orçamentário inicial de implantação e um valor mensal de custeio. No CEO, o valor depende da quantidade de especialidades e faixa mínima de procedimentos por especialidade para as quais foi habilitado (Herkratz et al., 2016).

Assim como no Reino Unido, a porta de entrada preferencial é pela APS, que é também responsável por ações preventivas e de promoção da saúde, com destaque para o trabalho em equipe multidisciplinar desenvolvido na Saúde da Família (Pucca, et al., 2015). Os serviços de laboratório de prótese dentária são majoritariamente prestados por laboratórios privados contratados. Existem ainda unidades odontológicas móveis e fluviais para populações de áreas mais remotas, atenção à pessoa com fissura labiopalatina e ao câncer bucal, ações de vigilância e promoção da saúde, como a fluoretação das águas de abastecimento público e inquéritos populacionais nacionais a cada dez anos. Os serviços de urgência e emergência vão desde a atenção primária aos hospitais (Cayetano et al., 2019; Pucca et al., 2015).

No NHS, os tratamentos odontológicos são realizados por clínicas e profissionais da rede privada credenciados, que podem funcionar inteira ou parcialmente pelo NHS (NHS, 2021; Sinclair et al., 2019). Para a população que não se enquadra nos critérios de gratuidade, há acesso a tratamento de urgência e co-pagamento dos demais tratamentos por desembolso direto, seguro privado odontológico ou pago pelos empregadores (Neumann \& Quiñonez, 2014; NHS, 2021). Quando o NHS começou, o atendimento odontológico era gratuito, mas as cobranças foram rapidamente introduzidas, e, na Escócia e Irlanda do Norte, atualmente, os usuários pagam até $80 \%$ dos custos (Cylus et al., 2015). As circunstâncias históricas do contexto pós-guerra favoreceram compromissos iniciais de manter a gratuidade dos serviços frente aos fortes impactos sociais do conflito, contudo, à medida que a situação se alterou, as pressões contrárias resultaram em sucessivas reformas (Filippon et al., 2016).

O usuário entra no NHS pela APS e os serviços especializados, de média e alta complexidade, são oferecidos nos hospitais. Destacam-se três estratégias de promoção da saúde bucal, criadas no RU entre 1999 e 2001, o National Healthy Schools, o Sure Start e o Brushing for life. O primeiro estimula práticas e atitudes desejáveis em saúde por meio da cultura escolar, contudo faltam ações explícitas de educação em saúde bucal. Já o Sure Start é um programa que fornece apoio aos pais e comunidade local com serviços de educação e saúde para famílias com crianças menores de 5 anos e o Brushing for Life é destinado às crianças de áreas mais vulneráveis e sem fluoretação das águas. Fornece também escovas e creme dental às famílias do Sure Start. Assim como em diversos países o acesso é limitado e, com frequência, associado a usuários de maior escolaridade e renda (Passalacqua et al., 2011). Iniciativas nacionais também compõem esse espectro, como o Programa Childsmile na Escócia (Kidd et al.,2020).

Quanto à fluoretação das águas de abastecimento público, no RU, apenas 11\% da população está coberta, com cerca de 6 milhões de pessoas beneficiadas apenas na Inglaterra (Chestnutt, 2013; OHF, 2020; NHS, 2021). Na Escócia, País de Gales e Irlanda do Norte não há um sistema de fluoretação artificial. A população da Escócia historicamente apresenta condições de saúde bucal ruins, e desde 2005 o país tem desenvolvido algumas iniciativas para crianças e idosos (Cylus et al., 2015). No Brasil, cerca de $76 \%$ da população é beneficiada com a fluoretação das águas, porém o monitoramento apresenta problemas e permanecem desigualdades regionais (Moimaz et al., 2020; Narvai \& Frazão, 2017). Estudo mais recente em municípios com mais de 50 mil habitantes mostrou uma cobertura populacional de 78,6\% para o país, entretanto, as regiões Sul e Sudeste apresentam as maiores taxas, e também são as regiões onde há maior vigilância da fluoretação (Roncalli et al., 2012).

E por fim, o financiamento e a gestão dos sistemas também apresentam diferenças importantes. As ações de saúde no RU são financiadas principalmente pelo governo, e os gastos per capita chegaram, em 2017, a US\$ 3.858 por habitante e a 9,6\% do PIB, sendo que cerca de $80 \%$ desse valor foi gasto público (WHO, 2018). No NHS, o financiamento é proveniente do setor público, a maior parte de impostos do Tesouro Nacional e uma pequena parcela do Sistema de Seguridade Social (Giovanella \& 
Stegmüller, 2014; Nicoletti \& Faria, 2017). Há uma estrutura centralizada integrada com organização regionalizada de serviços e a alocação de recursos obedece a necessidades populacionais e especificidades regionais (Giovanella et al., 2018). O Departamento de Saúde aloca fundos na Inglaterra, e são concedidos subsídios em bloco à Irlanda do Norte, Escócia e País de Gales. As administrações descentralizadas estabelecem políticas de saúde para a Irlanda do Norte, Escócia e País de Gales, enquanto a política de saúde do NHS Inglaterra é decidida pelo governo do Reino Unido diretamente (Cylus et al., 2015). Os fundos públicos contribuem com 30\% para o custo total da saúde bucal e os usuários com os outros 70\% (Sinclair et al., 2019). Estudo sobre a população idosa apontou que apenas 39,7\% do financiamento é governamental, 50,9\% por desembolso direto e 9,5\% por seguro odontológico privado (Allin et al., 2020).

A odontologia é uma prática liberal, e, desde os anos de 1990, parte do pagamento dos dentistas do NHS é feito por captação, e, a partir de 2006, recebem também por unidades de atividade odontológica (UDA) concluídas (Cylus et al., 2015). Essa reforma foi considerada uma das mudanças mais significativas na história do NHS, pois os dentistas passaram a ser contratados diretamente pelos prestadores através da UDA e foram incluídas metas (Tickle, 2012). Entre os objetivos da reforma estavam a melhoria do acesso, remuneração e condições de trabalho, bem como a redução das intervenções e mais tempo livre para o dentista atuar na prevenção. Entretanto, estudos apontam que eles não foram alcançados na prática, e a melhor forma de pagamento dos dentistas, se por capitação ou procedimento, segue como uma questão em disputa nos países (Chestnutt et al., 2009; Hill et al., 2020; Holmes et al., 2015). Além disso, apesar do aumento geral de dentistas registrados no RU, há relatos de serviços do NHS fechados pela dificuldade de provimento em algumas áreas do país, dentre outros motivos, pela remuneração considerada baixa (Davda et al., 2020).

No Brasil, a saúde também é financiada por meio de impostos gerais, contribuições sociais, desembolso direto e gastos dos empregadores (Figueiredo et al., 2018). O financiamento do SUS é proveniente de impostos das três esferas de governo, com mínimo de participação estabelecido por lei, de respectivamente, $12 \%$ para os estados, 15\% para os municípios, e, no caso da União, o percentual deve corresponder ao valor aplicado no exercício financeiro anterior, acrescido da variação do PIB (Figueiredo et al., 2018). O Brasil apresenta maior gasto privado do que público que o Reino Unido, e o subfinanciamento do SUS ficou ainda mais agravado devido às medidas de austeridade fiscal adotadas desde 2016, sobretudo a Emenda Constitucional 95 (EC 95/16), a qual estipulou um teto para a despesa primária da União e o congelamento do gasto em saúde por 20 anos (Figueiredo et al., 2018; Oliveira et al., 2021). O financiamento nominal da saúde bucal obteve aumento significativo após 2004, com o lançamento do Brasil Sorridente (Chaves et al., 2017). Houve aumento crescente do repasse federal nos anos de 2003 a 2010, seguido de um período de manutenção até 2016, e redução dos valores a partir de 2017 (Chaves et al., 2017; Rossi et al., 2019).

A maioria dos profissionais do SUS são remunerados através de salário, com algumas iniciativas de incorporação de pagamento por desempenho (Gabriel et al., 2020). Na APS, desde a entrada da saúde bucal no ano 2000, a carga horária de toda a equipe era de 40 horas semanais, com foco no vínculo e acompanhamento contínuo dos profissionais aos usuários, suas famílias e território (Brasil, 2011). Contudo, desde 2017, a nova Política Nacional de Atenção Básica vem flexibilizando os modelos de equipes, e em 2020, o MS permitiu composições de equipe de saúde bucal com 20 e 30 horas semanais (Brasil, 2017; Brasil, 2020). A atuação simultânea do dentista no setor público e privado é uma questão que merece aprofundamento nas duas realidades. Verifica-se tanto no SUS, quanto no NHS, que parte dos profissionais recém-formados consideram o setor público como um posto de trabalho temporário, para ganhar experiência antes do setor privado, enquanto parte valoriza o "espírito do serviço público" e se identifica com os princípios orientadores dos sistemas universais, ou ainda, valoriza a estabilidade do emprego público (Almeida et al., 2021; Araújo et al., 2021; Gallagher et al., 2009). No caso brasileiro, a saturação do mercado de trabalho em diversos locais, o alto número de faculdades e a ampliação das vagas no SUS, principalmente na atenção primária, são fatores a serem considerados (Araújo et al., 2021; Gabriel et al., 2020). 
A gestão do SUS é de comando único em cada esfera de governo (federal, estadual e municipal), sendo o Ministério da Saúde responsável pelo nível federal (Paim, 2018). A gestão nacional da política fica a cargo da Coordenação Geral de Saúde Bucal, e existem coordenações ou áreas técnicas responsáveis pela gestão dos serviços nos níveis estadual e municipal. A maior parte é gerida e ofertada pelo município, havendo alguns serviços de atenção especializada de gestão estadual (4,3\%), enquanto cabe ao MS, além do financiamento, a formulação de diretrizes e portarias regulamentadoras (Herkratz et al., 2016).

No Reino Unido, a regulação do sistema de saúde ocorre por legislação nacional e pelas agências especializadas independentes, o National Institute for Health and Care Excellence (NICE), o Monitor, e o Health Quality Commission (CQC) (Giovanella \& Stegmüller, 2014). Embora as recomendações do NICE sejam oficialmente apenas para a Inglaterra, existem acordos com a Irlanda do Norte, Escócia e País de Gales, e as respectivas administrações decidem como utilizá-las, tendo também órgãos e agências reguladoras próprias (Ferreira \& Mendes, 2018; Gallagher \& Eaton, 2015). Na atenção primária, desde 2006, os dentistas do NHS precisam cumprir metas pactuadas, e têm sido testadas estratégias de monitoramento e avaliação de desempenho dentro dos contratos, com governança mais flexível em relação ao órgão nacional (Gallagher \& Eaton, 2015).

Apesar da expansão dos serviços, ainda há muito a ser feito no Brasil. Verificam-se insuficiências na oferta, barreiras de acesso, desigualdades regionais, problemas na implementação como baixo financiamento, gestão inadequada, falta de manutenção dos insumos e equipamentos odontológicos, além de muitos locais manterem a perspectiva da odontologia curativa e tradicional, com alta prevalência de exodontias (Aquilante \& Aciole, 2015; Scarparo et al., 2015). Contudo, o reconhecimento da saúde como direito e expansão dos serviços públicos representam avanços, principalmente considerando que o Brasil é o único país capitalista periférico da América Latina que adotou um modelo de sistema público universal, e cerca de $75 \%$ da população depende do sistema público (Giovanella et al., 2018; Santos, 2018).

O RU, por sua vez, apresenta elevados indicadores de melhoria nas condições de saúde bucal da população nos últimos 50 anos, entretanto, desigualdades entre as classes sociais permanecem (Hui et al., 2020; Sinclair et al., 2019). Em algumas áreas do País de Gales, por exemplo, o número de dentistas é insuficiente e as crianças de baixa renda têm piores condições de saúde bucal (Cylus et al., 2015). Na faixa etária dos idosos, encontra-se maior dificuldade para tratamento daqueles institucionalizados ou quando são pacientes especiais (Hui et al., 2020). Observa-se redução no percentual de adultos e crianças que visitaram o dentista nos últimos 24 meses quando se compara os valores de 2014 e 2018 (Quadro 2). Chama atenção o componente condicional adotado nas últimas reformas dos contratos odontológicos, de 2009 a 2016, que restringe o acesso de usuários a determinados tratamentos até que cumpram medidas preventivas. Essa é uma estratégia alinhada às medidas de austeridade $\mathrm{e}$ discurso de eficiência, bastante questionável do ponto de vista social e da universalidade preconizada desde a origem do NHS (Laverty \& Harris, 2018).

\section{Considerações Finais}

Em ambos os países de sistema universal, a saúde é um direito constitucional, entretanto o acesso gratuito às ações de saúde bucal pelo NHS é restrito a um público específico, com serviços ofertados essencialmente por profissionais e clínicas do setor privado, enquanto o SUS possui serviços na atenção primária, especializada e hospitalar, com acesso universal e gratuito, de oferta pública, ainda que a cobertura seja insuficiente e com problemas de implementação. Cabe destacar que o funcionamento do Estado de bem-estar social britânico e as diferenças nos níveis de garantia e proteção social mínimas entre o Reino Unido e o Brasil são historicamente diferentes, assim como as realidades epidemiológica, de desenvolvimento econômico e social e gasto público em saúde também distintos, com indicadores mais desfavoráveis na situação brasileira.

A opção pela oferta pública e universal na saúde bucal, incluindo a atenção especializada, foi uma escolha relativamente recente no Brasil, que converge com os objetivos do SUS de reduzir as iniquidades e reorientar o modelo de atenção à saúde. Os profissionais do NHS apresentam uma lógica predominante da odontologia de mercado, e as sucessivas reformas aprofundaram 
a liberalização. As medidas de austeridade, assim como no Brasil, afastam cada vez mais o sistema de saúde dos seus princípios originários e norteadores, de universalidade, equidade e integralidade. Há presença do setor público e privado nos dois casos, sendo a regulação do setor privado e da formação de novos profissionais por parte do governo brasileiro bastante inferior quando comparada ao Reino Unido. Esses elementos necessitam de aprofundamento em novos estudos.

A análise comparativa realizada apresenta limitações quanto ao escopo da revisão e do próprio uso de dados secundários. Contudo, mesmo de forma panorâmica, representa uma contribuição para o estudo de políticas de saúde, ao atualizar, sintetizar e discutir as principais características da atenção à saúde bucal em dois sistemas universais de saúde, e auxiliar na identificação de elementos que contribuem para o avanço da oferta pública da atenção odontológica. A crise sanitária decorrente da pandemia do novo coronavírus em curso desde 2020 não foi objeto desta análise, todavia reconhece-se o seu impacto nos dois países e nos respectivos sistemas de saúde, com dados mais graves no Brasil. Recomenda-se monitoramento contínuo das políticas e dos sistemas de serviços de saúde, e aprofundamento dos achados em cada um dos componentes analisados através de revisões sistemáticas, bem como novos estudos comparativos.

\section{Agradecimentos}

Esse estudo foi desenvolvido no âmbito do Programa de Iniciação Científica (PIBIC-Voluntário) da Universidade Federal do Recôncavo da Bahia (UFRB), em parceria interinstitucional através do Projeto Construção das propostas de financiamento para a saúde no Legislativo, suas mudanças e relações com o Sistema de Saúde: uma análise comparativa entre

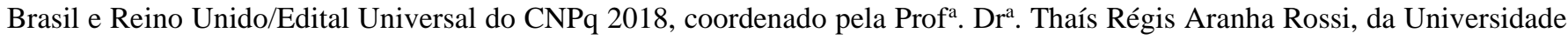
do Estado da Bahia (UNEB).

\section{Referências}

Allin, S., Farmer, J., Quiñonez, C., Peckham, A., Marchildon, G., Panteli, D., Henschke, C., Fattore, G., Lamloum, D., Holden, A. C. L., \& Rice, T. (2020). Do health systems cover the mouth? Comparing dental care coverage for older adults in eight jurisdictions. Health Policy, 124(9), 998-1007. https://doi.org/10.1016/j.healthpol.2020.06.015

Almeida, D. C. L. de, Fadel, C. B., \& Silva Júnior, M. F. (2021). Mercado de trabalho público: percepção de formandos em Odontologia de uma universidade pública. Research, Society and Development, 10(8), e49110817702. https://doi.org/10.33448/rsd-v10i8.17702

Aquilante, A. G., \& Aciole, G. G. (2015). Building a "Smiling Brazil"? Implementation of the Brazilian National Oral Health Policy in a health region in the State of São Paulo. Cadernos de Saúde Pública, 31(1), 82-96. https://doi.org/10.1590/0102-311X00193313

Araújo, J. P. C. de, Firmeza, L. M. D., Almeida, M. E. L. de, \& Teixeira, A. K. M. (2021). Perfil dos egressos do curso de Odontologia da Universidade Federal do Ceará e suas percepções acerca do mercado de trabalho. Revista Da ABENO, 21(1), 1073. https://doi.org/10.30979/rev.abeno.v21i1.1073

Brasil. Lei $\mathrm{n}^{\circ}$ 11.889, de 24 de dezembro de 2008. (2008). Regulamenta o exercício das profissões de Técnico em Saúde Bucal - TSB e de Auxiliar em Saúde Bucal - ASB. http://www.planalto.gov.br/ccivil_03/_ato2007-2010/2008/lei/111889.htm

Brasil. Ministério da Saúde. Secretaria de Atenção à Saúde. Secretaria de Vigilância em Saúde. Departamento de Atenção Básica. Coordenação geral de Saúde Bucal. (2012). Projeto SB Brasil 2010: pesquisa nacional de saúde bucal: resultados principais. Brasília (DF): Ministério da Saúde; 2011. http://189.28.128.100/dab/docs/geral/projeto_sb2010_relatorio_final.pdf

Brasil. Portaria GM/MS no 2.488, de 21 de outubro de 2011. (2011). Aprova a Política Nacional de Atenção Básica, estabelecendo a revisão de diretrizes e normas para a organização da Atenção Básica, para a Estratégia Saúde da Família (ESF) e o Programa de Agentes Comunitários de Saúde (PACS). https://bvsms.saude.gov.br/bvs/saudelegis/gm/2011/prt2488_21_10_2011.html

Brasil. Portaria GM/MS nº 2.436, de 21 de setembro de 2017. (2017). Aprova a Política Nacional de Atenção Básica, estabelecendo a revisão de diretrizes para a organização da Atenção Básica, no âmbito do Sistema Único de Saúde (SUS). Diário Oficial da União, 22 Set 2017. https://bvsms.saude.gov.br/bvs/saudelegis/gm/2017/prt2436_22_09_2017.html

Brasil. Ministério da Saúde. Secretaria de Atenção Primária à Saúde. Portaria nº 60, de 26 de novembro de 2020. (2020). Define as regras de validação das equipes e serviços da Atenção Primária à Saúde, para fins da transferência dos incentivos financeiros federais de custeio. https://www.in.gov.br/en/web/dou//portaria-n-60-de-26-de-novembro-de-2020-290853767

Brasil. (2020). Sistema e-Gestor Atenção Básica. Relatórios Públicos. https://egestorab.saude.gov.br/

Cayetano, M. H., Carrer, F. C. de A., Gabriel, M., Martins, F. C., \& Pucca Jr., G. A. (2019). Política Nacional de Saúde Bucal Brasileira (Brasil Sorridente): Um resgate da história, aprendizados e futuro. Universitas Odontológica, 38(80). https://doi.org/10.11144/Javeriana.uo38-80.pnsb 
Chaves, S. C. L., Almeida, A. M. F. de L., Rossi, T. R. A., Santana, S. F. de, Barros, S. G. de, \& Santos, C. M. L. (2017). Política de Saúde Bucal no Brasil 2003-2014: cenário, propostas, ações e resultados. Ciência \& Saúde Coletiva, 22(6), 1791-1803. https://doi.org/10.1590/1413-81232017226.18782015

Chestnutt, I. G. (2013). Addressing oral health inequalities in the United Kingdom - the impact of devolution on population-based fluoride policy. British Dental Journal, 215(1), 11-12. https://doi.org/10.1038/sj.bdj.2013.626

Chestnutt, I. G., Davies, L., \& Thomas, D. R. (2009). Practitioners' perspectives and experiences of the new National Health Service dental contract. British Dental Journal, 206(9), E18-E18. https://doi.org/10.1038/sj.bdj.2009.354

Conselho Federal de Odontologia. (2021). Estatísticas. http://cfo.org.br/website/estatisticas/quantidade-geral-de-entidades-e-profissionais-ativos/

Coughlan, J., \& Shah, S. (2020). The impact of Brexit on oral health. British Dental Journal, 229(9), 622-626. https://doi.org/10.1038/s41415-020-2278-z

Csikar, J. I., Bradley, S., Williams, S. A., Godson, J. H., \& Rowbotham, J. S. (2009). Dental therapy in the United Kingdom: part 4. Teamwork - is it working for dental therapists? British Dental Journal, 207(11), 529-536. https://doi.org/10.1038/sj.bdj.2009.1104

Cylus, J., Richardson, E., Findley, L., Longley, M., O’Neill, C., \& Steel, D. (2015). United Kingdom: Health System Review. Health Systems in Transition, 17(5), 1-126. http://www.ncbi.nlm.nih.gov/pubmed/27049966

Davda, L. S., Radford, D. R., Scambler, S., \& Gallagher, J. E. (2020). Profiles of registrant dentists and policy directions from 2000 to 2020. BDJ Open, 6(1), 26. https://doi.org/10.1038/s41405-020-00054-1

Downer, M. C., Drugan, C. S., \& Blinkhorn, A. S. (2005). Dental caries experience of British children in an international context. Community Dental Health, 22(2), 86-93. http://www.ncbi.nlm.nih.gov/pubmed/15984133

Ferreira, M. R. J., \& Mendes, A. N. (2018). Mercantilização nas reformas dos sistemas de saúde alemão, francês e britânico. Ciência \& Saúde Coletiva, 23(7), 2159-2170. https://doi.org/10.1590/1413-81232018237.12972018

Figueiredo, J. O., Prado, N. M. de B. L., Medina, M. G., \& Paim, J. S. (2018). Gastos público e privado com saúde no Brasil e países selecionados. Saúde Em Debate, 42(spe2), 37-47. https://doi.org/10.1590/0103-11042018s203

Filippon, J., Giovanella, L., Konder, M., \& Pollock, A. M. (2016). A “liberalização” do Serviço Nacional de Saúde da Inglaterra: trajetória e riscos para o direito à saúde. Cadernos de Saúde Pública, 32(8). https://doi.org/10.1590/0102-311X00034716

Frazão, P., \& Narvai, P. C. (2017). Water fluoridation in Brazilian cities at the first decade of the 21 st century. Revista de Saúde Pública, 51. https://doi.org/10.1590/s1518-8787.2017051006372

Gabriel, M., Cayetano, M. H., Chagas, M. M., Araújo, M. E. de, Dussault, G., Pucca Junior, G. A., \& Almeida, F. C. S. de. (2020). Mecanismos de ingresso de dentistas no SUS: uma agenda prioritária para o fortalecimento do Brasil Sorridente. Ciência \& Saúde Coletiva, 25(3), 859-868. https://doi.org/10.1590/141381232020253.14532018

Gallagher, J. E., Clarke, W., Eaton, K. A., \& Wilson, N. H. F. (2009). A question of value: a qualitative study of vocational dental practitioners' views on oral healthcare systems and their future careers. Primary Dental Care: Journal of the Faculty of General Dental Practitioners (UK), 16(1), 29-37. https://doi.org/10.1308/135576109786994550

General Dental Council. (2017). General Dental Council Reports. https://www.gdc-uk.org/

General Dental Council. (2019). Registration statistical report. https://www.gdc-uk.org/docs/default-source/registration-reports/gdc-registration-statisticalreport-2019---final-30-09-2020.pdf

General Dental Council. (2013). The scope of your practice. www.gdc-uk.org/information-standards-guidance/standards-and-guidance/scope-of-practice

Giovanella, L., \& Stegmüller, K. (2014). The financial crisis and health care systems in Europe: universal care under threat? Trends in health sector reforms in Germany, the United Kingdom, and Spain. Cadernos de Saúde Pública, 30(11), 2263-2281. https://doi.org/10.1590/0102-311X00021314

Giovanella, L., Mendoza-Ruiz, A., Pilar, A. de C. A., Rosa, M. C. da, Martins, G. B., Santos, I. S., Silva, D. B., Vieira, J. M. de L., Castro, V. C. G. de, Silva, P. O. da, \& Machado, C. V. (2018). Sistema universal de saúde e cobertura universal: desvendando pressupostos e estratégias. Ciência \& Saúde Coletiva, 23(6), 1763-1776. https://doi.org/10.1590/1413-81232018236.05562018

Herkratz, F. J., Vieira, J. M. R., Vettore, M. V., Rebelo, M. A. B., Figueiredo, N., \& Castro, P. H. D. F. de. (2016). Atenção secundária em saúde bucal no Brasil: distribuição da oferta e sua influência na acessibilidade e equidade. In N. Figueiredo, P. S. A. de Goes, \& P. J. de L. Martelli (Eds.), Os caminhos da saúde bucal no Brasil: um olhar quali e quanti sobre os Centro de Especialidade Odontológicas (CEO) no Brasil (pp. 37-48). Editora UFPE. https://www.researchgate.net/publication/319847849_Atencao_secundaria_em_saude_bucal_no_Brasil_distribuicao_da_oferta_e_sua_influencia_na_acessibil idade_e_equidade

Hobdell, M., Petersen, P. E., Clarkson, J., \& Johnson, N. (2003). Global goals for oral health 2020. International Dental Journal, 53(5), 285-288. https://doi.org/10.1111/j.1875-595x.2003.tb00761.x

Hui, A., Latif, A., Hinsliff-Smith, K., \& Chen, T. (2020). Exploring the impacts of organisational structure, policy and practice on the health inequalities of marginalised communities: Illustrative cases from the UK healthcare system. Health Policy, 124(3), 298-302. https://doi.org/10.1016/j.healthpol.2020.01.003

Instituto Brasileiro de Geografia e Estatística. (2021). Países. https://paises.ibge.gov.br/\#/mapa

Kidd, J. B., McMahon, A. D., Sherriff, A., Gnich, W., Mahmoud, A., Macpherson, L. M., \& Conway, D. I. (2020). Evaluation of a national complex oral health improvement programme: a population data linkage cohort study in Scotland. BMJ Open, 10(11), e038116. https://doi.org/10.1136/bmjopen-2020-038116 
Lenza, M. M. de O., Rodrigues, L. G., Lenza, M. G., Prado, M. M. do, \& Silva, R. F. (2019). Limites de atuação da equipe de saúde bucal em um panorama global. Revista Brasileira de Odontologia Legal, 6(3), 59-72. https://doi.org/10.21117/rbol.v6i3.271

Leyssen, W., Clark, R. K. F., Gallagher, J. E., \& Radford, D. R. (2013). Developing professional status: an investigation into the working patterns, working relationships and vision for the future of UK clinical dental technicians. British Dental Journal, 214(2), E3-E3. https://doi.org/10.1038/sj.bdj.2013.55

Masood, M., Mnatzaganian, G., \& Baker, S. R. (2019). Inequalities in dental caries in children within the UK: Have there been changes over time? Community Dentistry and Oral Epidemiology, 47(1), 71-77. https://doi.org/10.1111/cdoe.12426

Miranda, P. R., \& Azevedo, M. L. N. De. (2020). Fies e Prouni na expansão da educação superior brasileira: políticas de democratização do acesso e/ou de promoção do setor privado-mercantil? Educação Formação, 5(3), e1421. https://doi.org/10.25053/redufor.v5i15set/dez.1421

Moimaz, S. A. S., Santos, L. F. P. dos, Saliba, T. A., Saliba, N. A., \& Saliba, O. (2020). Vigilância em saúde: fluoretação das águas de abastecimento público em 40 municípios do estado de São Paulo, Brasil. Ciência \& Saúde Coletiva, 25(7), 2653-2662. https://doi.org/10.1590/1413-81232020257.03972018

Morita, M. C., Uriarte Neto, M., Fontanella, V. R. C., \& Haddad, A. E. (2021). The unplanned and unequal expansion of Dentistry courses in Brazil from 1856 to 2020. Brazilian Oral Research, 35. https://doi.org/10.1590/1807-3107bor-2021.vol35.0009

National Health Service. (2021). Who is entitled to free NHS dental treatment in England? https://www.nhs.uk/common-health-questions/dental-health/who-isentitled-to-free-nhs-dental-treatment-in-england/

Neumann D. G. \& Quiñonez C. A. (2014). A comparative analysis of oral health care systems in the United States, United Kingdom, France, Canada, and Brazil. NCOHR Working Paper Series, 1(2). http://dx.doi.org/10.13140/RG.2.1.2224.2726

Nicoletti, M. A., \& Faria, T. D. M. (2017). Análise comparativa dos sistemas de saúde brasileiro e britânico na atenção básica. Infarma - Ciências Farmacêuticas, 29(4), 313-327. https://doi.org/10.14450/2318-9312.v29.e4.a2017.pp313-327

Oliveira B. F., Cruz, F. P. Soares, I. M. \& Oliveira, H. R. H. (2021). Privatização do Sistema Único de Saúde (SUS): Uma revisão da literatura a partir de artigos científicos da base de dados da Scielo. Research, Society and Development, 10(5):e9610514676.

Oral Health Foundation. (2020). Water fluoridation policy. https://www.dentalhealth.org/water-fluoridation-policy

Paim, J. S. (2018). Sistema Único de Saúde (SUS) aos 30 anos. Ciência \& Saúde Coletiva, 23(6), 1723-1728. https://doi.org/10.1590/141381232018236.09172018

Passalacqua, A., Reeves, A. O., Newton, T., Hughes, R., Dunne, S., Donaldson, N., \& Wilson, N. (2012). An assessment of oral health promotion programmes in the United Kingdom. European Journal of Dental Education: Official Journal of the Association for Dental Education in Europe, 16(1), e19-26. https://doi.org/10.1111/j.1600-0579.2010.00667.x

Peres, M. A., Macpherson, L. M. D., Weyant, R. J., Daly, B., Venturelli, R., Mathur, M. R., Listl, S., Celeste, R. K., Guarnizo-Herreño, C. C., Kearns, C., Benzian, H., Allison, P., \& Watt, R. G. (2019). Oral diseases: a global public health challenge. Lancet (London, England), 394(10194), 249-260. https://doi.org/10.1016/S0140-6736(19)31146-8

Pucca, G. A., Gabriel, M., de Araujo, M. E., \& de Almeida, F. C. S. (2015). Ten Years of a National Oral Health Policy in Brazil: Innovation, Boldness, and Numerous Challenges. Journal of Dental Research, 94(10), 1333-1337. https://doi.org/10.1177/0022034515599979

Rossi, T. R. A., Lorena Sobrinho, J. E. de, Chaves, S. C. L., \& Martelli, P. J. de L. (2019). Crise econômica, austeridade e seus efeitos sobre o financiamento e acesso a serviços públicos e privados de saúde bucal. Ciência \& Saúde Coletiva, 24(12), 4427-4436. https://doi.org/10.1590/1413-812320182412.25582019

Roncalli, A. G., Côrtes, M. I. de S., \& Peres, K. G. (2012). Perfis epidemiológicos de saúde bucal no Brasil e os modelos de vigilância. Cadernos de Saúde Pública, 28(suppl), s58-s68. https://doi.org/10.1590/S0102-311X2012001300007

Santos, N. R. dos. (2018). SUS 30 anos: o início, a caminhada e o rumo. Ciência \& Saúde Coletiva, 23(6), 1729-1736. https://doi.org/10.1590/141381232018236.06092018

Scarparo, A., Zermiani, T. C., Ditterich, R. G., \& Pinto, M. H. B. (2015). Impacto da Política Nacional de Saúde Bucal - Programa Brasil Sorridente - sobre a provisão de serviços odontológicos no Estado do Rio de Janeiro. Cadernos Saúde Coletiva, 23(4), 409-415. https://doi.org/10.1590/1414-462X201500040153

Sinclair, E., Eaton, K. A., \& Widström, E. (2019). The healthcare systems and provision of oral healthcare in European Union member states. Part 10: comparison of systems and with the United Kingdom. British Dental Journal, 227(4), 305-310. https://doi.org/10.1038/s41415-019-0661-4

Souza, L. E. P. F., \& Bahia, L. (2014). Componentes de um Sistema de Serviços de Saúde: população, infraestrutura, organização, prestação de serviços, fianciamento e gestão. In Paim, J. S. \& Almeida Filho, N. (Eds.), Saúde Coletiva - teoria e prática (pp. 49-68). Medbook Editora Científica Ltda.

Steele, J. G., Treasure, E. T., O'Sullivan, I., Morris, J., \& Murray, J. J. (2012). Adult Dental Health Survey 2009: transformations in British oral health 19682009. British Dental Journal, 213(10), 523-527. https://doi.org/10.1038/sj.bdj.2012.1067

Tanaka, O. Y., \& Oliveira, V. E. de. (2007). Reforma(s) e estruturação do Sistema de Saúde Britânico: lições para o SUS. Saúde e Sociedade, 16(1), 7-17. https://doi.org/10.1590/S0104-12902007000100002

Tickle, M. (2012). Revolution in the provision of dental services in the UK. Community Dentistry and Oral Epidemiology, 40(Suppl 2), 110-116. https://doi.org/10.1111/j.1600-0528.2012.00729.x

Warmling, C. M., Rosa, E. K. da, Pezzato, L. M., \& Toassi, R. F. C. (2016). Competências de Auxiliares e Técnicos de Saúde Bucal e o Vínculo com o Sistema Único de Saúde. Trabalho, Educação e Saúde, 14(2), 575-592. https://doi.org/10.1590/1981-7746-sip00116 
Research, Society and Development, v. 10, n. 10, e185101018444, 2021

(CC BY 4.0) | ISSN 2525-3409 | DOI: http://dx.doi.org/10.33448/rsd-v10i10.18444

Watt, R. G., Daly, B., Allison, P., Macpherson, L. M. D., Venturelli, R., Listl, S., Weyant, R. J., Mathur, M. R., Guarnizo-Herreño, C. C., Celeste, R. K., Peres, M. A., Kearns, C., \& Benzian, H. (2019). Ending the neglect of global oral health: time for radical action. The Lancet, 394(10194), 261-272. https://doi.org/10.1016/S0140-6736(19)31133-X

Whittemore, R., \& Knafl, K. (2005). The integrative review: updated methodology. Journal of Advanced Nursing, 52(5), 546-553. https://doi.org/10.1111/j.1365-2648.2005.03621.x

World Health Organizations. (2020). Global Health Observatory data repository: Dentistry personnel. https://apps.who.int/gho/data/node.main.HWF2

World Health Organizations. (2018). Public Spending on Health: A Closer Look at Global Trends. https://www.who.int/health_financing/documents/healthexpenditure-report-2018/en/

Zanetti, C. H. G., Oliveira, J. A. A. de, \& Mendonça, M. H. M. de. (2012). Divisão do trabalho odontológico em perspectiva: desafio de interpretar as competências dos técnicos. Trabalho, Educação e Saúde, 10(2), 195-222. https://doi.org/10.1590/S1981-77462012000200002 